\title{
Predicting device failure after percutaneous repair of functional mitral regurgitation in advanced heart failure: Implications for patient selection
}

\author{
Davide Stolfo $^{\mathrm{a}, *, 1}$, Antonio De Luca ${ }^{\mathrm{a}, 1}$, Gaetano Morea ${ }^{\mathrm{a}}$, Marco Merlo ${ }^{\mathrm{a}}$, Giancarlo Vitrella ${ }^{\mathrm{a}}$, Thomas Caiffa ${ }^{\mathrm{a}}$, \\ Giulia Barbati ${ }^{\mathrm{b}}$, Serena Rakar ${ }^{\mathrm{a}}$, Renata Korcova ${ }^{\mathrm{a}}$, Andrea Perkan ${ }^{\mathrm{a}}$, Bruno Pinamonti ${ }^{\mathrm{a}}$, Aniello Pappalardo ${ }^{\mathrm{c}}$, \\ Alessandra Berardini ${ }^{\mathrm{d}}$, Elena Biagini ${ }^{\mathrm{d}}$, Francesco Saia ${ }^{\mathrm{d}}$, Francesco Grigioni ${ }^{\mathrm{d}}$, \\ Claudio Rapezzi ${ }^{\mathrm{d}}$, Gianfranco Sinagra ${ }^{\mathrm{a}}$ \\ a Cardiovascular Department, Division of Cardiology, Azienda Sanitaria Universitaria Integrata di Trieste, Italy \\ ${ }^{\mathrm{b}}$ Biostatistics Unit, Department of Medical Sciences, University of Trieste, Trieste, Italy \\ c Cardiovascular Department, Division of Cardiac Surgery, Azienda Sanitaria Universitaria Integrata di Trieste, Italy \\ d Institute of Cardiology, University Hospital "S. Orsola-Malpighi”, Bologna, Italy
}

\section{A R T I C L E I N F O}

\section{Article history:}

Received 27 October 2017

Received in revised form 11 December 2017

Accepted 2 January 2018

Available online 6 January 2018

Keywords:

Heart failure

MitraClip

Mitral annulus

Mitral regurgitation

\begin{abstract}
A B S T R A C T
Background: Patients with heart failure (HF) and severe symptomatic functional mitral regurgitation (FMR) may benefit from MitraClip implantation. With increasing numbers of patients being treated the success of procedure becomes a key issue. We sought to investigate the pre-procedural predictors of device failure in patients with advanced HF treated with MitraClip.

Methods: From April 2012 to November 2016, 76 patients with poor functional class (NYHA class III-IV) and severe left ventricular (LV) remodeling underwent MitraClip implantation at University Hospitals of Trieste and Bologna (Italy). Device failure was assessed according to MVARC criteria. Patients were subsequently followed to additionally assess the patient success after 12 months.

Results: Mean age was $67 \pm 12$ years, the mean Log-EuroSCORE was $23.4 \pm 16.5 \%$, and the mean LV end-diastolic volume index and ejection fraction (EF) were $112 \pm 33 \mathrm{ml} / \mathrm{m}^{2}$ and $30.6 \pm 8.9 \%$, respectively. At short-term evaluation, device failure was observed in 22 (29\%) patients. Univariate predictors of device failure were LVEF, LV and left atrial volumes and anteroposterior mitral annulus diameter. Annulus dimension (OR $1.153,95 \% \mathrm{CI}$ $1.002-1.327, p=0.043$ ) and LV end-diastolic volume (OR 1.024, 95\% CI 1.000-1.049, $p=0.049$ ) were the only variables independently associated with the risk of device failure at the multivariate model.

Conclusions: Pre-procedural anteroposterior mitral annulus diameter accurately predicted the risk of device failure after MitraClip in the setting of advanced HF. Its assessment might aid the selection of the best candidates to percutaneous correction of FMR.
\end{abstract}

(c) 2018 Elsevier B.V. All rights reserved.

\section{Introduction}

Functional mitral regurgitation (FMR) is a common finding in patients with heart failure (HF) and left ventricular (LV) dysfunction, and is independently associated with a poor prognosis [1]. In recent years percutaneous correction of MR with the MitraClip system (Abbott Vascular, Abbott Park, Illinois, USA) has been established as an alternative treatment option for surgical high-risk patients with degenerative and functional MR $[2,3]$. Worldwide experience reports high procedural success rates and favorable clinical outcomes in patients with systolic

\footnotetext{
* Corresponding author at: Cardiovascular Department, University Hospital of Trieste Via Valdoni 7, 34149 Trieste, Italy.

E-mail address: davide.stolfo@asuits.sanita.fvg.it (D. Stolfo).

${ }^{1}$ Coauthorship: these two Authors contributed equally to this paper as first Author.
}

HF and FMR [2,4]. However, despite good early results, the risk of device failure after MitraClip is emerging as a major issue. For instance, realworld registries have reported a 1-year recurrence rate of FMR $>20 \%$ $[5,6,7]$. This claims the need of valuable tools for selecting the optimal candidates to MitraClip. Therefore, in the present study we sought to identify pre-procedural predictors of device failure, then exploring its effects on outcomes in patients with advanced heart failure (HF), FMR and importantly remodeled left ventricle (LV).

\section{Methods}

From April 2012 to December 2016, all the patients undergoing percutaneous mitral valve repair with MitraClip system (Abbott Vascular, Abbott Park, Illinois, USA) in two third-level Italian referral centers for HF (University Hospital of Trieste and University Hospital "St. Orsola-Malpighi" of Bologna) were consecutively included in a prospective registry. Patients with FMR and advanced HF were considered eligible for the present 
Table 1

Baseline characteristics of the overall study cohort and divided according to device failure.

\begin{tabular}{|c|c|c|c|c|}
\hline & $\begin{array}{l}\text { Global } \\
n=75\end{array}$ & $\begin{array}{l}\text { Device failure } \\
\mathrm{n}=22(29 \%)\end{array}$ & $\begin{array}{l}\text { Device success } \\
\mathrm{n}=53(71 \%)\end{array}$ & $p$ \\
\hline \multicolumn{5}{|c|}{ a) Clinical and demographics - baseline } \\
\hline Age - years & $67 \pm 12$ & $63 \pm 14$ & $68 \pm 11$ & 0.109 \\
\hline $\mathrm{BMI}-\mathrm{kg} / \mathrm{m}^{2}$ & $25 \pm 4$ & $24 \pm 4$ & $25 \pm 4$ & 0.695 \\
\hline Male sex - \% & 80 & 73 & 83 & 0.310 \\
\hline NYHA IV - \% & 23 & 27 & 21 & 0.665 \\
\hline $\mathrm{BNP}-\mathrm{pg} / \mathrm{ml}^{\mathrm{a}}$ & $502[304 ; 1011]$ & $644[253 ; 1097]$ & $502[314 ; 865]$ & 0.733 \\
\hline Pro-BNP $-\mathrm{pg} / \mathrm{ml}^{\mathrm{b}}$ & $5291[1569 ; 8184]$ & $3499[1555 ; 9962]$ & $5479[1606 ; 8184]$ & 0.667 \\
\hline Furosemide - mg & $125[50 ; 250]$ & $112[75 ; 250]$ & $138[50 ; 256]$ & 0.648 \\
\hline Logistic EuroSCORE - \% & $22 \pm 18$ & $21 \pm 14$ & $23 \pm 19$ & 0.649 \\
\hline Ischemic CMP - \% & 51 & 46 & 53 & 0.561 \\
\hline Hypertension - \% & 59 & 46 & 64 & 0.134 \\
\hline Diabetes - \% & 37 & 36 & 38 & 0.911 \\
\hline COPD - \% & 12 & 9 & 13 & 0.617 \\
\hline $\mathrm{CKD}-\%$ & 73 & 77 & 72 & 0.619 \\
\hline $\mathrm{GFR}-\mathrm{ml} / \mathrm{min} / 1.73 \mathrm{~m}^{2}$ & $57 \pm 28$ & $55 \pm 27$ & $57 \pm 29$ & 0.803 \\
\hline $\mathrm{ICD}-\%$ & 69 & 82 & 64 & 0.131 \\
\hline CRT $-\%$ & 37 & 59 & 28 & 0.012 \\
\hline No. clips $\geq 2(\%)$ & 61 & 64 & 60 & 0.477 \\
\hline A FIB/atrial flutter - \% & 40 & 54 & 34 & 0.098 \\
\hline Beta blockers - \% & 79 & 68 & 83 & 0.153 \\
\hline ACEi/ARBs - \% & 76 & 86 & 72 & 0.102 \\
\hline MRAs - \% & 67 & 82 & 60 & 0.073 \\
\hline ASA - \% & 54 & 48 & 57 & 0.452 \\
\hline OAT - \% & 44 & 52 & 40 & 0.359 \\
\hline \multicolumn{5}{|l|}{ Echocardiography - baseline } \\
\hline LVEDVi $-\mathrm{ml} / \mathrm{m}^{2}$ & $114 \pm 35$ & $130 \pm 39$ & $108 \pm 31$ & 0.011 \\
\hline LVESVi $-\mathrm{ml} / \mathrm{m}^{2}$ & $76 \pm 29$ & $88 \pm 36$ & $71 \pm 25$ & 0.016 \\
\hline LVEF - \% & $31 \pm 8$ & $27 \pm 9$ & $32 \pm 8$ & 0.024 \\
\hline RV dysfunction - \% & 55 & 59 & 53 & 0.620 \\
\hline Restrictive pattern - \% & 77 & 82 & 75 & 0.524 \\
\hline LA AREAi $-\mathrm{mm} / \mathrm{m}^{2}$ & $18 \pm 5$ & $19 \pm 8$ & $17 \pm 4$ & 0.118 \\
\hline $\mathrm{TR} \geq 2+-\%$ & 20 & 18 & 21 & 0.800 \\
\hline $\mathrm{SPAP}-\mathrm{mm} \mathrm{Hg}$ & $51 \pm 13$ & $53 \pm 15$ & $50 \pm 12$ & 0.460 \\
\hline Annulus diameter - mm & $37 \pm 5$ & $39 \pm 4$ & $36 \pm 4$ & 0.025 \\
\hline Tenting area $-\mathrm{cm}^{2}$ & $2.47 \pm 0.76$ & $2.73 \pm 0.91$ & $2.36 \pm 0.66$ & 0.049 \\
\hline Vena contracta width $-\mathrm{mm}$ & $7.6 \pm 2.2$ & $8.0 \pm 2.4$ & $7.4 \pm 2.1$ & 0.391 \\
\hline EROa (Pisa method) $-\mathrm{mm}^{2}$ & $39 \pm 16$ & $40 \pm 16$ & $39 \pm 17$ & 0.793 \\
\hline \multicolumn{5}{|l|}{ b) Echocardiography - early follow-up } \\
\hline $\mathrm{LVEDVi}-\mathrm{ml} / \mathrm{m}^{2}$ & & $125 \pm 53$ & $95 \pm 26$ & 0.003 \\
\hline LVESVi $-\mathrm{ml} / \mathrm{m}^{2}$ & & $96 \pm 45$ & $65 \pm 22$ & 0.001 \\
\hline LVEF - \% & & $24 \pm 8$ & $31 \pm 8$ & 0.003 \\
\hline RV dysfunction - \% & & 61 & 41 & 0.140 \\
\hline $\mathrm{TR} \geq 2+-\%$ & & 56 & 26 & 0.026 \\
\hline $\mathrm{SPAP}-\mathrm{mm} \mathrm{Hg}$ & & $51 \pm 17$ & $40 \pm 12$ & 0.003 \\
\hline Maximum gradient $-\mathrm{mm} \mathrm{Hg}$ & & $9.3 \pm 4.3$ & $9.5 \pm 4.1$ & 0.864 \\
\hline Mean gradient $-\mathrm{mm} \mathrm{Hg}$ & & $3.7 \pm 2.9$ & $3.3 \pm 2.1$ & 0.661 \\
\hline MVA (PHT method) $-\mathrm{cm}^{2}$ & & $3.3 \pm 1.1$ & $2.1 \pm 0.5$ & $<0.001$ \\
\hline \multicolumn{5}{|l|}{ c) Clinical - 12 month follow-up } \\
\hline$>1$ grade NYHA improvement - $\%$ & & 50 & 77 & 0.05 \\
\hline NYHA I - \% & & 0 & 2 & 0.006 \\
\hline NYHA II - \% & & 42 & 70 & \\
\hline NYHA III - \% & & 33 & 28 & \\
\hline NYHA IV - \% & & 25 & 0 & \\
\hline
\end{tabular}

Values are expressed as the mean \pm SD or median with interquartile range, and as a percentage (\%).

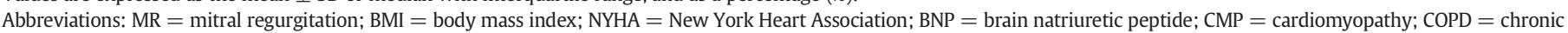

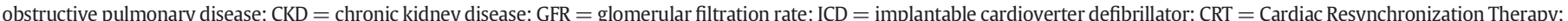

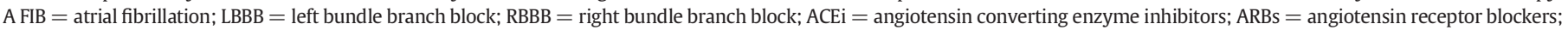

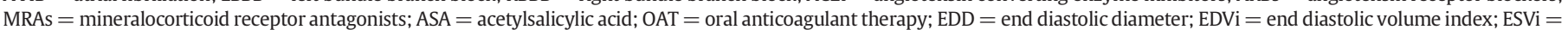

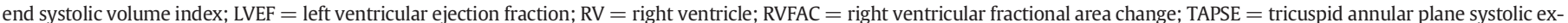
cursion; LA = left atrium; TR = tricuspid regurgitation; SPAP = Systolic Pulmonary Artery Pressure; EROa = effective regurgitant orifice area; MVA = Mitral Valve Area.

a Data available for the series of Trieste.

b Data available for the series of Bologna.

study. Inclusion criteria were high grade FMR $(\geq 3+)$, severe HF symptoms (New York Heart Association - NYHA - class III-IV) and significant left ventricle (LV) dysfunction (ejection fraction - EF - <40\%) refractory to currently recommended HF therapy, including Cardiac Resynchronization Therapy (CRT) if clinically indicated [8]. Indications to percutaneous treatment were given by the multidisciplinary Heart Team of the referral center, according to current guidelines [8,9]. EVEREST II eligibility criteria were used as reference for patient selection $[2,3]$.
Before the procedure patients underwent complete clinical evaluation, laboratory tests, ECG, transthoracic and transesophageal echocardiography in order to assess feasibility or rule out any contraindications [9]. Logistic European System for Cardiac Operative Risk Evaluation (EuroSCORE) was calculated to estimate the surgical risk.

Device success at early follow-up (median time 4 months, range 2-6) was primarily investigated and defined according to Mitral Valve Academic Research Consortium (MVARC) criteria. Procedural success was also assessed (see supplementary appendix 
for full definitions) [10]. Procedure-related complications were collected. Patients underwent a short-term complete clinical and echocardiographic follow-up. Exploratory outcome analysis was performed by evaluating patient success at 12 months (see supplementary appendix for complete definition ) [10]. Follow-up ended on the 30th of September 2017 or at the time of death. Information regarding the end-points was obtained from the patients, their physician or the registers of death of the municipalities of residence. The local institutional review board approved the study protocol. All patients gave written informed consent for data collection and analysis.

Echocardiograms were recorded on digital media storage at the echocardiographic core laboratory of our Institutions and analyzed offline. FMR was graded by a multiparametric approach, based on transthoracic and transesophageal echocardiography, according to the current recommendations of the American Society of Echocardiography [11] and European Association of Cardiovascular Imaging [12]. Structural measurements were systematically performed. In particular the anteroposterior annulus diameter was assessed by 2D transesophageal echocardiography in the mid-esophageal $120^{\circ}$ view and the tenting area was measured in mid-systole as the area between the mitral annulus plane and the mitral leaflet body [12]. LV dimensions and systolic and diastolic function were assessed according to international guidelines [13,14]. Specifically, LV volumes and LVEF were calculated by Simpson's biplane method, left atrial (LA) size was assessed by end-systolic biplane volume. All volumes were indexed according to body surface area. RV systolic dysfunction was assessed in accordance with current guidelines by estimation or RV fractional area change (RV FAC) or tricuspid annular plane systolic excursion (TAPSE) and defined by a RV FAC $<35 \%$ or a TAPSE $<17 \mathrm{~mm}$ [13]. Systolic Pulmonary Artery Pressure (SPAP) was estimated from the peak flow velocity at continuous-wave Doppler of the tricuspid regurgitation jet using systolic transtricuspidal pressure gradient by the modified Bernoulli equation and the addition of estimated right atrial pressure [15]. All measurements were obtained from the mean of 3 beats (patients in sinus rhythm) or 5 beats (atrial fibrillation).

\subsection{Statistical analysis}

Summary statistics of clinical and laboratory variables were expressed as mean \pm standard deviation (for normally distributed continuous variables) or median and interquartile range (for non-normally distributed continuous variables), or counts and percentage (for nominal variables). Cross-sectional comparisons between groups were made by the ANOVA test on Gaussian distributed continuous variables, using the Brown-Forsythe statistic when the assumption of equal variances did not hold, or the non-parametric Median test when necessary. The Chi-square or Fisher's exact tests were calculated for discrete variables. Pearson's Rho correlation coefficients between echocardiographic LV and mitral measures were computed in order to have a ranking of the strength of the association. Univariate logistic regression models were estimated to evaluate associations between pre-procedural parameters and MitraClip failure at follow-up. Since the limited number of events and the small sample size, a penalized logistic multivariable regression model was estimated starting from the list of significant variables at univariate analysis, by using Firth's penalized likelihood [16]. A $p$ value $<0.05$ was considered to indicate statistical significance. The IBM SPSS software, version 19 and the R statistical package version 3.1.2 (with library "logistf") were used for the analysis.

\section{Results}

\subsection{Study population and procedural data}

A total of 76 patients fulfilling the inclusion criteria underwent MitraClip procedure. Implantation was successful in 99\% of cases. The single patient with acute procedural failure (failure to grasp both mitral leaflets due to extreme loss of coaptation) was not reassessed at followup and died 4 months after procedure (pre-procedural annulus diameter $40 \mathrm{~mm}$ ). The remaining 75 patients had a MR grade $\leq 2+$ at the end of procedure. One clip was implanted in 30 patients (40\%), 2 clips in 42 patients (55\%) and 3 clips in 4 patients (5\%). Fifteen procedure-related complications occurred in 11 patients (see Supplementary Table 2 for the complete list of procedure-related complications). No patient died during procedure. The preoperative clinical and echocardiographic features of the study population are reported in Table 1 . Mean age was 67 \pm 12 years, $80 \%$ were males, $51 \%$ had $\mathrm{HF}$ of ischemic etiology. All the patients were in NYHA class III (77\%) or IV (23\%). Surgical risk was globally high (Log EuroSCORE $22 \pm 18 \%$ ). Mean Left Ventricular End Diastolic Volume index (LVEDVi), Left Ventricular End Systolic Volume index (LVESVi) and LVEF were $114 \pm 35 \mathrm{ml} / \mathrm{m}^{2}, 76 \pm 29 \mathrm{ml} / \mathrm{m}^{2}$ and $31 \pm$ $8 \%$, respectively while RV function was impaired in 41 subjects (55\%). Mean SPAP was $51 \pm 13 \mathrm{~mm} \mathrm{Hg}$. Twenty-one patients (28\%) had a pre-procedural LVEF $\leq 25 \%$. Mean anteroposterior annulus diameter and tenting area were $37 \pm 5 \mathrm{~mm}$ and $2.47 \pm 0.76 \mathrm{~cm}^{2}$, respectively. Finally, patients were all on optimal medical therapy and 28 (37\%) had previously undergone CRT implantation. At discharge (median time 4 days, range 2-6) severe MR was documented in only 5 cases (7\%), whereas $83 \%$ had none or mild MR.

\subsection{Correlation analysis}

Correlations between echocardiographic metrics are shown in Supplementary Table 3. LV volumes and LVEF were strongly correlated to each other. Of note, annulus diameter and tenting area were not significantly correlated with measures of MR severity (i.e. vena contracta width and EROa), but both showed a significant moderate correlation with LVEDVi ( $r=0.33$ vs annulus diameter and $r=0.50$ vs tenting area, $p<0.05$ for both), LVESVi ( $r=0.37$ vs annulus diameter and $r=$ 0.46 vs tenting area, $p<0.05$ for both) and $\operatorname{LVEF}(r=-0.25$ vs annulus diameter and $r=-0.29$ vs tenting area, $p<0.05$ for both).

\subsection{Predictors of device failure}

Follow-up information was available for 75 patients. Device failure occurred in 22 patients ( $29 \%$ of the study population) and procedural failure in 26 (34\%). As showed in Table 1, at pre-procedural assessment patients experiencing device failure more frequently had a CRT implanted compared to patients with device success (59\% vs $28 \%, p=$ 0.012); LV remodeling was more severe (LVEDVi $130 \pm 39 \mathrm{ml} / \mathrm{m}^{2}$ vs $108 \pm 31 \mathrm{ml} / \mathrm{m}^{2}, p=0.011$ and LVESVi $88 \pm 36 \mathrm{ml} / \mathrm{m}^{2}$ vs $71 \pm$ $25 \mathrm{ml} / \mathrm{m}^{2}, p=0.016$ ) and systolic function was significantly worse (LVEF $27 \pm 9 \%$ vs $32 \pm 8 \%, p=0.024$ ). Concerning mitral structural abnormalities, the anteroposterior annulus diameter ( $39 \pm 4$ vs $36 \pm$ $4 \mathrm{~mm}, p 0.025)$ and the tenting area $\left(2.73 \pm 0.91\right.$ vs $2.36 \pm 0.66 \mathrm{~cm}^{2}$, $p$ 0.049) were significantly larger in the device failure group.

In order to identify pre-procedural parameters associated with device failure at short-term follow-up a multivariate model was created starting from the variables associated with device failure at univariate analysis (Table 2). The anteroposterior annulus diameter measured at transesophageal echocardiography was independently related with the likelihood of device failure (odds ratio - OR - 1.153, 95\% Confidence Interval $-\mathrm{CI}-1.002-1.327, p=0.043$ ) along with LVEDVi (OR 1.024, $95 \%$ CI $1.000-1.049, p=0.049)$. Fig. 1 shows the incidence of device failure at follow-up in patients with a pre-procedural annulus diameter $\geq 35 \mathrm{~mm}$ vs those $<35 \mathrm{~mm}$ ( $35 \%$ vs $11 \%$ respectively; $p=0.04$ ).

3.4. Evolution of the main echocardiographic measures, clinical features and outcomes during follow-up according to device success

As reported in Table 1, echocardiographic re-evaluation at early follow-up confirmed the difference in LV volumes and LVEF between the two groups (Table 1b). Fig. 2 shows the trends of the main echocardiographic metrics at follow-up: interestingly, patients with device success had a significant reduction in LVEDVi (from $108 \pm 31$ to $95 \pm$

\section{Table 2}

Univariate and multivariable Cox regression analysis: independent predictors of device failure.

\begin{tabular}{|c|c|c|c|c|c|c|}
\hline \multirow[b]{2}{*}{ Variable } & \multicolumn{3}{|c|}{ Univariate } & \multicolumn{3}{|c|}{ Multivariate } \\
\hline & OR & $95 \% \mathrm{CI}$ & $p$ & OR & $95 \% \mathrm{CI}$ & $p$ \\
\hline LVEDVi $\left(\mathrm{ml} / \mathrm{m}^{2}\right)$ & 1.019 & $1.003-1.035$ & 0.018 & 1.024 & $1.000-1.049$ & 0.049 \\
\hline LVESVi $\left(\mathrm{ml} / \mathrm{m}^{2}\right)$ & 1.020 & $1.002-1.038$ & 0.025 & & & \\
\hline LVEF (\%) & 0.927 & $0.868-0.992$ & 0.028 & & & \\
\hline LAVi & 1.020 & $1.000-1.040$ & 0.043 & & & \\
\hline $\begin{array}{l}\text { Mitral annulus } \\
\text { diameter (mm) }\end{array}$ & 1.142 & $1.013-1.287$ & 0.031 & 1.153 & $1.002-1.327$ & 0.046 \\
\hline
\end{tabular}

Abbreviations: $\mathrm{OR}=$ odds ratio; $\mathrm{CI}=$ Confidence Interval; $\mathrm{EDVi}=$ end diastolic volume index; ESVi = end systolic volume index; LAVI = left atrial volume index; LVEF = left ventricular ejection fraction. Odds ratio estimation is referred to every unit increase. Odds ratio estimation is referred to every unit increase or decrease for continuous variables. 


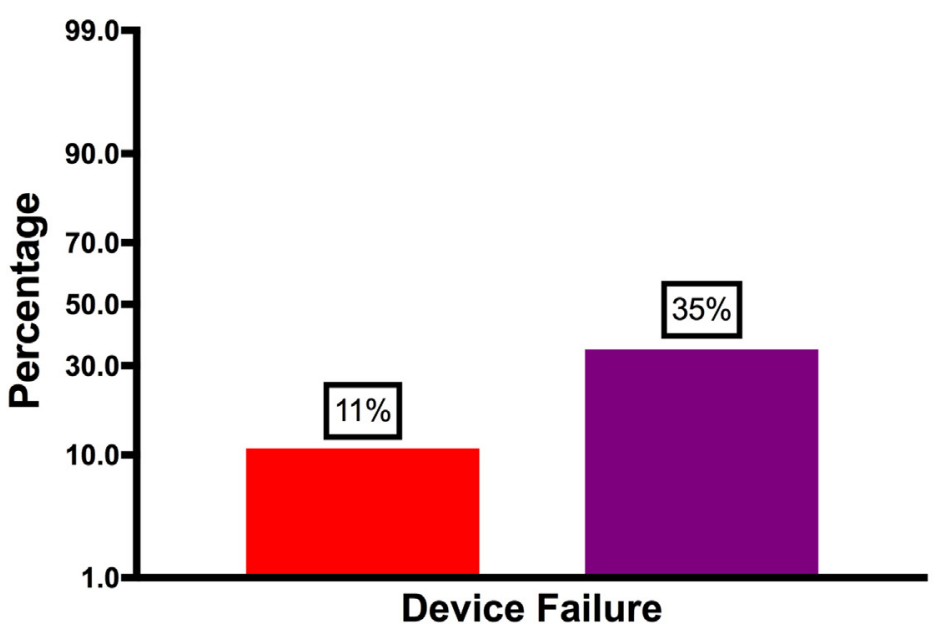

Annulus $<35 \mathrm{~mm}$

Annulus $\geq 35 \mathrm{~mm}$

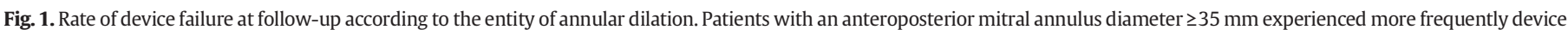
failure $(p=0.04)$

$26 \mathrm{ml} / \mathrm{m}^{2} ; p<0.001$ ) and $\mathrm{sPAP}$ ( from $50 \pm 12$ to $40 \pm 12 \mathrm{~mm} \mathrm{Hg} ; p<$ 0.001 ), while those with device failure were characterized by a significant worsening in LVESVi (from $88 \pm 36$ to $96 \pm 45 \mathrm{ml} / \mathrm{m}^{2} ; p=$ 0.030 ) and LVEF ( $27 \pm 9$ to $24 \pm 8 \% ; p=0.043$ ). There was no
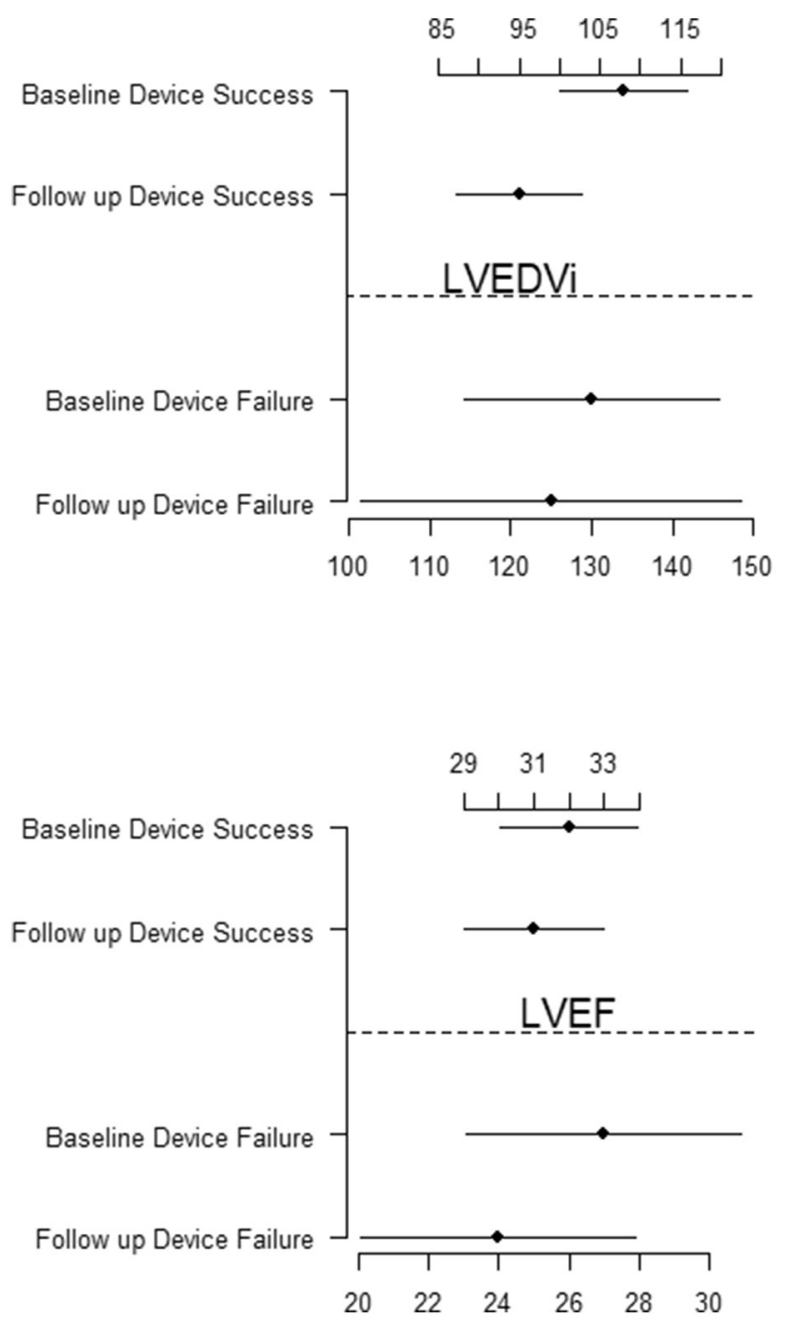

between-groups difference in transvalvular gradient, while the estimated Mitral Valve Area by Pressure Half Time (PHT) method was significantly smaller in the device success group $\left(2.1 \pm 0.5\right.$ vs $3.3 \pm 1.1 \mathrm{~cm}^{2}$, $p<0.001$ ).
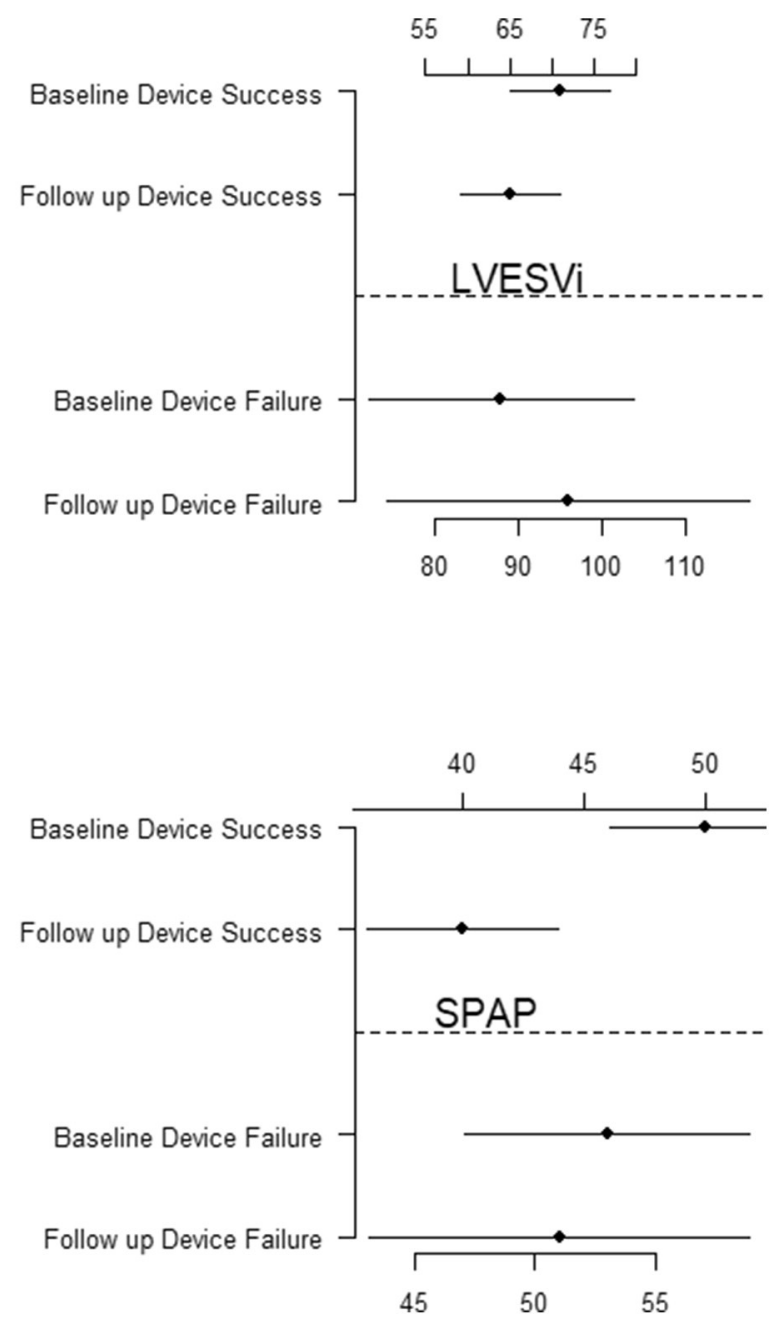

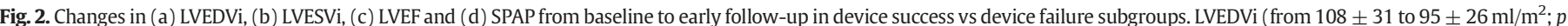

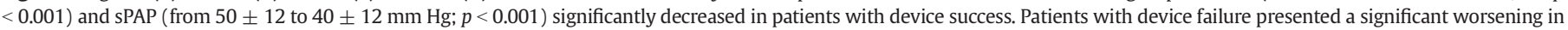

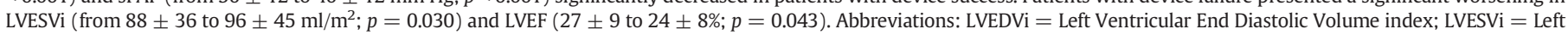
Ventricular End Systolic Volume index; LVEF = left ventricular ejection fraction; SPAP = Systolic Pulmonary Artery Pressure. 
Device success was associated with a higher rate of $\geq 1$ NYHA class improvement at 12-months of follow-up as compared with patients experiencing device failure group ( $77 \%$ vs $50 \%, p=0.05$, Table $1 c$, Supplementary Fig. 1). Furthermore, device success was the only predictor of patient success (OR 35.579; 95\% CI 4.680-301.751) at 1 year, with a rate of patient success of $64 \%$ vs $4.5 \%$ in patients with device failure, $p<0.001$ (Supplementary Fig. 2).

\section{Discussion}

The main finding of our study is that among the several potential clinical and echocardiographic indicators of LV remodeling and geometric distortion of the mitral apparatus, anteroposterior diameter of the mitral annulus and LVEDVi showed the strongest association with device failure and might be particularly useful for the selection of the optimal candidates to percutaneous treatment of FMR in high-risk populations with advanced HF and severe LV remodeling. This is of particular interest in this setting of patients since the failure of the device after MitraClip confirmed its negative impact on 1-year outcome.

Randomized studies have demonstrated the safety and the short and mid-term efficacy of percutaneous correction of MR with MitraClip in patients deemed at high risk for surgery $[17,18]$. Whereas EVEREST II enrolled predominantly patients with primary MR, large registries on MitraClip therapy describe a different "real-life" scenario. Indeed, MitraClip candidates in clinical practice are typically older, with a more advanced HF and affected mostly by FMR. Although the procedure has been confirmed to be safe, with few adverse events and positive results $[3,5,6,19,20]$, in a variable proportion of patients the prognosis remains poor. In previous studies highly symptomatic HF, largely remodeled LV and very depressed LVEF have been correlated with a lower event-free survival after MitraClip [21,22], raising a concern in the identification of optimal candidates to implantation. Although the worse outcome can be partially justified by the advanced status of the disease by itself, the degree of residual MR has been demonstrated significantly impacting the prognosis of patients who underwent MitraClip [23]. In large observational registries acute procedural failure exhibited the strongest prognostic effect on mid-term survival [19,24]. However, even when optimal results are obtained acutely after MitraClip implantation, MR may recur during follow-up in a significant proportion of patients. De Bonis et al. reported a 4 year freedom from MR $\geq 3+$ in $75 \%$ of patients with initial good procedural result [25]. Similarly, the incidence of MR $>2+$ at follow-up was close to $20 \%$ in both Swiss and ACCESS-EU cohorts $[5,24]$. Nevertheless, few studies are available describing the impact of device failure following MitraClip as defined by recently introduced MVARC criteria, in particular in patients with advanced HF [10, $19,24,26]$. In our cohort of patients with FMR and large LV remodeling, device failure occurred in $29 \%$ of cases, negatively impacting on clinical outcomes with a very low rate of persistent patient success after 1 year. It is possible that the high incidence of device failure reported in our cohort reflects a more advanced population compared to that enrolled in the aforementioned registries. Consistently, the success of procedure was also associated with larger improvement in symptoms and in the hemodynamic profile.

Based on these assumptions, recognizing who will more likely experience device failure among patients eligible for the procedure becomes critical to minimize the risk of procedural futility and to optimize the benefit for the single patient, especially in the advanced HF setting. Few studies have focused on the identification of patients at risk for early procedural failure. A restricted posterior leaflet motion, expression of asymmetric tethering, was associated with failing mitral repair along with severe pre-procedural pulmonary hypertension in a series of 91 patients with advanced systolic HF and FMR [27]. In our series patients showing an early device failure presented the echocardiographic features of a more severe $\mathrm{LV}$ remodeling. The only variables independently associated with the risk of device failure at multivariable analysis were the diameter of the mitral annulus measured at transesophageal echocardiography and the LVEDVi. This is the principal finding of our study, since to the best of our knowledge a significant relation between the pre-procedural annulus geometry and the risk of early MitraClip failure in patients with secondary MR has not been previously reported.

In FMR, the massive geometric distortion of the mitral annulus is due to the progression of LV remodeling. Recently, the changes in the annulus size after MitraClip implantation, but not the entity of dilatation assessed pre-procedurally, showed a moderate correlation with residual MR during follow-up in a heterogeneous cohort of degenerative and functional MR [28]. In our series we found that parameters assessing the geometry of the mitral annulus (anteroposterior diameter and tenting area) were significantly correlated with measures of LV remodeling, rather than with the indicators of MR severity. Undersized annuloplasty associated with the edge-to-edge Alfieri procedure during surgery correction of FMR reduced the incidence of repair failure in patients with excessive tethering [29]. Markers of LV and annulus remodeling are the major predictors of repair failure in conventional surgery as well [8]. As MitraClip can be considered the percutaneous adaptation of the surgical edge-to-edge technique lacking mitral annuloplasty, a higher risk of procedural failure for cases presenting with extensive LV remodeling and annulus deformation should be taken into account in the selection of candidates for MitraClip. Strategies combining MitraClip and percutaneous annuloplasty devices or alternatively the complete transcatheter mitral valve replacement might become in the next future the best approach for similar patients [30]. Moreover, measures of mitral annular distortion may simply identify a subpopulation of patients with advanced disease who do not benefit from MitraClip implantation and should be quickly referred to mechanical circulatory supports/heart transplantation, if not contraindicated, or end of life care. Noteworthy, in our samples the anteroposterior diameter of the annulus $\geq 35 \mathrm{~mm}$, that was similar to the previously reported threshold for failing of surgical repair in ischemic FMR [12], discriminated patients with high rate of device failure ( $35 \%$ vs $11 \%$ in those with annulus diameter $<35 \mathrm{~mm}, p=$ 0.04 ; Fig. 1). Finally, in future studies 3D preprocedural evaluation of the whole annulus geometry and direct intraprocedural 3D assessment of residual area and geometric changes after MitraClip delivery should be systematically assessed and correlated to early and later indicators of procedural failure and worse outcomes.

\subsection{Limitations}

Several limitations need to be acknowledged. As all observational studies, it suffers from the common bias of different selection criteria and treatments. The data were collected from two different third-level referral centers for HF. Although the two samples were largely comparable in terms of clinical characteristics, echocardiographic metrics and treatments with the exception of biomarker evaluation since two different molecules were assessed in the two centers (BNP in Trieste Hospital and proBNP in Bologna Hospital), a potential bias due to the variability in local practice and clinical selection criteria across institutions is still possible. The lack of a single core Echo-lab for imaging assessment can be considered a further limitation. The small size of our cohort might limit the impact of our observations. However, only few small studies investigated the setting of end-stage HF patients undergoing MitraClip, and predictors of device failure in this specific subset are lacking. Another potential limit concerns the timing of follow-up that was arbitrary chosen. There is no general agreement on the optimal timing for the echocardiographic evaluation of stable effects of MitraClip on secondary MR.

Preprocedural measures of the mitral annulus were only performed by conventional 2D-transesophageal echocardiography and anteroposterior diameter was considered according to larger expert consensus. Preliminary data have recently reported the association of acute reduction in anteroposterior mitral valve geometry assessed by 3Dtransesophageal echocardiography with durability of MitraClip procedure in FMR [31]. In our cohort 3D reconstruction of the mitral apparatus 
was not available for all the study patients. However, the measurement of the annulus diameter by 2D approach is feasible, more reproducible and widely accessible, thus appropriate for screening the patients eligible to percutaneous correction of FMR. Finally, it is known that the echocardiographic quantification of residual MR after MitraClip suffers from major limitations due to the presence of a double-orifice. Observational studies reported the superiority of Cardiac Magnetic Resonance for the determination of post-procedural regurgitation. Unfortunately, the majority of our patients carried non-MRI-compatible implanted devices (ICD and/or CRT), precluding this option.

\section{Conclusions}

Our study confirmed the negative impact of device failure after MitraClip in patients with advanced LV remodeling and severe systolic HF while a stable correction of FMR exerted a positive impact in terms of symptom relief and outcomes. Therefore, the correct selection of patients eligible for the procedure becomes of primary importance. In the present series, the anteroposterior diameter of the mitral annulus emerged as a promising indicator of the risk of early device failure along with LVEDV, being the expression of a more advanced LV remodeling. Future studies including a systematic comprehensive assessment of the mitral apparatus geometry are warranted to clearly identify the optimal candidates for percutaneous correction of FMR, including combined leaflet and annular techniques, and the optimal timing of procedure.

\section{Disclosures}

None.

\section{Acknowledgments}

CRT Foundation of Trieste for the continuous support in research.

\section{Appendix A. Supplementary data}

Supplementary data to this article can be found online at https://doi. org/10.1016/j.ijcard.2018.01.009.

\section{References}

[1] D. Stolfo, M. Merlo, B. Pinamonti, et al., Early improvement of functional mitral regurgitation in patients with idiopathic dilated cardiomyopathy, Am. J. Cardiol. 115 (2015) 1137-1143

[2] O. Franzen, J. van der Heyden, S. Baldus, et al., MitraClip ${ }^{\circledR}$ therapy in patients with end-stage systolic heart failure, Eur. J. Heart Fail. 13 (2011) 569-576.

[3] T. Feldman, E. Foster, D.D. Glower, et al., Percutaneous repair or surgery for mitral regurgitation, N. Engl. J. Med. 364 (2011) 1395e1406.

[4] A. Auricchio, W. Schillinger, S. Meyer, et al., Correction of mitral regurgitation in nonresponders to cardiac resynchronization therapy by MitraClip improves symptoms and promotes reverse remodeling, J. Am. Coll. Cardiol. 58 (2011) 2183-2189.

[5] F. Maisano, O. Franzen, S. Baldus, et al., Percutaneous mitral valve interventions in the real world: early and 1-year results from the ACCESS-EU, a prospective, multicenter, nonrandomized post-approval study of the MitraClip therapy in Europe, J. Am. Coll. Cardiol. 62 (2013) 1052-1061.

[6] G. Nickenig, R. Estevez-Loureiro, O. Franzen, et al., Percutaneous mitral valve edgeto-edge repair: in-hospital results and 1-year follow-up of 628 patients of the 2011-2012 Pilot European Sentinel Registry, J. Am. Coll. Cardiol. 64 (2014) 875-884.

[7] N. Buzzatti, M. De Bonis, P. Denti, et al., What is a "good" result after transcatheter mitral repair? Impact of $2+$ residual mitral regurgitation, J. Thorac. Cardiovasc. Surg. 151 (2016) 88-96.
8] A. Vahanian, O. Alfieri, F. Andreotti, et al., Guidelines on the management of valvular heart disease (version 2012), Eur. Heart J. 33 (2012) 2451-2496.

[9] D.H. Bail, K. Doebler, The MitraClip System: a systematic review of indications, procedural requirements, and guidelines, Thorac. Cardiovasc. Surg. 62 (2014) 18-25.

[10] G.W. Stone, D.H. Adams, W.T. Abraham, et al., Clinical trial design principles and endpoint definitions for transcatheter mitral valve repair and replacement: part 2: endpoint definitions: a consensus document from the Mitral Valve Academic Research Consortium, J. Am. Coll. Cardiol. 66 (2015) 308-321.

[11] W.A. Zoghbi, M. Enriquez-Sarano, E. Foster, et al., Recommendations for evaluation of the severity of native valvular regurgitation with two-dimensional and Doppler echocardiography, J. Am. Soc. Echocardiogr. 16 (2003) 777-802.

[12] P. Lancellotti, L. Moura, L.A. Pierard, et al., European Association of Echocardiography recommendations for the assessment of valvular regurgitation. Part 2: mitral and tricuspid regurgitation (native valve disease), Eur. J. Echocardiogr. 11 (2010) 307-332.

[13] R.M. Lang, L.P. Badano, V. Mor-Avi, et al., Recommendations for cardiac chamber quantification by echocardiography in adults: an update from the American Society of Echocardiography and the European Association of Cardiovascular Imaging, J. Am. Soc. Echocardiogr. 28 (2015) 1-39.e14.

[14] S.F. Nagueh, O.A. Smiseth, C.P. Appleton, et al., Recommendations for the evaluation of left ventricular diastolic function by echocardiography: an update from the American Society of Echocardiography and the European Association of Cardiovascular Imaging, J. Am. Soc. Echocardiogr. 29 (4) (2016 Apr) 277-314.

[15] A. Milan, C. Magnino, F. Veglio, Echocardiographic indexes for the non-invasive evaluation of pulmonary hemodynamics, J. Am. Soc. Echocardiogr. 23 (2010) 224-225.

[16] D. Firth, Bias reduction of maximum likelihood estimates, Biometrika 80 (1993) 27-38.

[17] T. Feldman, M. Cilingiroglu, Percutaneous leaflet repair and annuloplasty for mitral regurgitation, J. Am. Coll. Cardiol. 57 (2011) 529-537.

[18] D.D. Glower, S. Kar, A. Trento, et al., Percutaneous mitral valve repair for mitral regurgitation in high-risk patients: results of the EVEREST II study, J. Am. Coll. Cardiol. 64 (2014) 172-181.

[19] M. Puls, E. Lubos, P. Boekstegers, et al., One-year outcomes and predictors of mortality after MitraClip therapy in contemporary clinical practice: results from the German transcatheter mitral valve interventions registry, Eur. Heart J. 37 (2016) 703-712.

[20] A. Berardini, E. Biagini, F. Saia, et al., Percutaneous mitral valve repair: the last chance for symptoms improvement in advanced refractory chronic heart failure? Int. J. Cardiol. 228 (2016) 191-197.

[21] D. Capodanno, M. Adamo, M. Barbanti, et al., Predictors of clinical outcomes after edge-to-edge percutaneous mitral valve repair, Am. Heart J. 170 (2015) 187-195.

[22] V. Rudolph, M. Huntgeburth, R.S. von Bardeleben, et al., Clinical outcome of critically ill, not fully recompensated, patients undergoing MitraClip therapy, Eur. J. Heart Fail. 16 (2014) 1223-1229.

[23] P.L. Whitlow, T. Feldman, W.R. Pedersen, et al., Acute and 12-month results with catheter-based mitral valve leaflet repair: the EVEREST II (Endovascular Valve Edge-to-Edge Repair) high risk study, J. Am. Coll. Cardiol. 59 (2012) 130-139.

[24] D. Sürder, G. Pedrazzini, O. Gaemperli, et al., Predictors for efficacy of percutaneous mitral valve repair using the MitraClip system: the results of the MitraSwiss registry, Heart 99 (2013) 1034-1040.

[25] M. De Bonis, E. Lapenna, N. Buzzatti, et al., Optimal results immediately after MitraClip therapy or surgical edge-to-edge repair for functional mitral regurgitation: are they really stable at 4 years? Eur. J. Cardiothorac. Surg. 50 (2016 Sep) 488-494.

[26] P.A. Grayburn, E. Foster, C. Sangli, et al., Relationship between the magnitude of reduction in mitral regurgitation severity and left ventricular and left atrial reverse remodeling after MitraClip therapy, Circulation 128 (2013) 1667-1674.

[27] M. Taramasso, P. Denti, A. Latib, et al., Clinical and anatomical predictors of MitraClip therapy failure for functional mitral regurgitation: single central clip strategy in asymmetric tethering, Int. J. Cardiol. 186 (2015) 286-288.

[28] J. Patzelt, Y. Zhang, H. Magunia, et al., Improved mitral valve coaptation and reduced mitral valve annular size after percutaneous mitral valve repair (PMVR) using the MitraClip system, Eur. Heart J. Cardiovasc. Imaging (2017 Aug 1), https://doi.org/ 10.1093/ehjci/jex173.

[29] M. De Bonis, E. Lapenna, G. La Canna, et al., Mitral valve repair for functional mitral regurgitation in end-stage dilated cardiomyopathy: role of the "edge-to-edge" technique, Circulation 112 (2005) I402-8.

[30] M.A. Acker, M.K. Parides, L.P. Perrault, et al, Mitral-valve repair versus replacement for severe ischemic mitral regurgitation, N. Engl. J. Med. 370 (2014 Jan 2) 23-32.

[31] R. Schueler, D. Momcilovic, M. Weber, et al., Acute changes of mitral valve geometry during interventional edge-to-edge repair with the MitraClip system are associated with midterm outcomes in patients with functional valve disease: preliminary results from a prospective single-center study, Circ. Cardiovasc. Interv. 7 (2014 Jun) 390-399. 